\title{
Eccentric topological properties of a graph associated to a finite dimensional vector space
}

https://doi.org/10.1515/mgmc-2020-0020

Received November 15, 2019; accepted March 04, 2020.

Abstract: A topological index is actually designed by transforming a chemical structure into a number. Topological index is a graph invariant which characterizes the topology of the graph and remains invariant under graph automorphism. Eccentricity based topological indices are of great importance and play a vital role in chemical graph theory. In this article, we consider a graph (nonzero component graph) associated to a finite dimensional vector space over a finite filed in the context of the following eleven eccentricity based topological indices: total eccentricity index; average eccentricity index; eccentric connectivity index; eccentric distance sum index; adjacent distance sum index; connective eccentricity index; geometric arithmetic index; atom bond connectivity index; and three versions of Zagreb indices. Relationship of the investigated indices and their dependency with respect to the involved parameters are also visualized by evaluating them numerically and by plotting their results.

\footnotetext{
*Corresponding author: Masood Ur Rehman, Department of Mathematics, Abbottabad University of Science and Technology, Abbottabad, Pakistan; e-mail: masoodqau27@gmail.com, masood@mail.ustc.edu.cn

Jia-Bao Liu, School of Mathematics and Physics, Anhui Jianzhu University, Hefei 230601, China; e-mail: liujiabaoad@163.com Imran Khalid, Center for Advanced Studies in Pure and Applied Mathematics, Bahauddin Zakariya University Multan, 60800, Pakistan; e-mail: imran.khalid@bzu.edu.pk

Mohammad Tariq Rahim, Department of Mathematics, Abbottabad University of Science and Technology, Abbottabad, Pakistan; e-mail: tariq.rahim@nu.edu.pk

Faisal Ali, Center for Advanced Studies in Pure and Applied Mathematics, Bahauddin Zakariya University Multan, 60800, Pakistan; e-mail: faisalali@bzu.edu.pk

Muhammad Salman, Department of Mathematics, The Islamia University of Bahawalpur, 63100, Pakistan;

e-mail: muhammad.salman@iub.edu.pk
}

Keywords: distance; degree; eccentricity; topological index

AMS Subject Classification Numbers: 15A27; 05C07; 05C12; 05C25; 05C69; 20E45

\section{Introduction}

Let a connected graph $G$ having the sets $V(G)$ and $E(G)$ as the vertex set and the edge set, respectively. Starting from a vertex $v$ and ending at a vertex $u$ in $G$, a shortest alternating sequence of vertices and edges without repetition of any vertex is known as a $v-u$ geodesic. The number of edges in a $v-u$ geodesic is denoted by $d(v, u)$, and is called the distance between $v$ and $u$ in $G$. The maximum distance of a vertex $v$ among its distances with all the vertices of $G$ is called the eccentricity of $v$, denoted by ecc $(v)$. The number $\operatorname{diam}(G)=\max _{v \in V(G)} \operatorname{ecc}(v)$ is the diameter of $G$. For any vertex $v$ of $G$, the number $D(v \mid G)=\sum_{u \in V(G)} d(v, u)$ is the distance number of $v$, and the number $d(v)$ is the degree of $v$.

An involvement of different graph theoretic tools/ parameters in the field of chemistry to deal with chemical structures is due to chemical graph theory. In chemicalgraph theory, an interesting sub field, namely cheminformatics, deals with chemical phenomenal "quantitative structureactivity/structure-property relationships" of chemical compounds. An emerging tool used, in the study of these phenomenal, is a topological index, which is invariant for chemical structures up to their symmetry (automorphism). Correlation of many physico-chemical properties like boiling point, stability, strain energy, etc. of chemical compounds in a chemical structure is due to its topological index (Bruckler et al., 2011; Deng et al., 2011; Klavžar and Gutman, 1996; Liu et al., 2019; Rucker and Rucker, 1999; Tang et al., 2019; Zheng et al., 2019; Zhang and Zhang, 1996). In this regard, the very first topological index was introduced by Wiener in 1947, and gave it name the path 
number (Wiener, 1947). This index is based on the concept of distance, mathematically, defined as:

$$
W(G)=\sum_{u, v \in V(G)} d(u, v)
$$

and called the Wiener index of a chemical structure/ graph $G$. Extending the study of topological index, based on the distance, many graph theorists introduced and studied various topological indices. Among these topological indices, a lot of researchers considered topological indices, defined using the eccentricity of each vertex, and produced remarkable investigations, such as: the average eccentricity of a graph and its subgraph was investigated in Dankelmann et al. (2004); the extremal properties of the average eccentricity and conjectures about the average eccentricity were obtained in Ilic (2012); lower and upper bounds of average eccentricity for trees were provided in Tang and Zhou (2012); the average eccentricity and standard deviation of Sierpinski graphs were established in Hinz and Parisse (2012); the eccentricity based ABC and geometric arithmetic indices for copper oxide networks are obtained in Imran et al. (2017); in Gao et al. (2016), results about the eccentric ABC index of linear polyene parallelogram benzenoid are investigated; the total eccentricity, average eccentricity, eccentricitybased Zagreb indices, eccentricity based atom bond connectivity $(\mathrm{ABC})$ and geometric arithmetic indices for oxide networks were found in Imran et al. (2018); eccentricity based topological indices of a cyclic octahedron structure were explored in Zahid et al. (2018). Furthermore, results regarding the average eccentricity index and eccentricity based geometric arithmetic index can be found in Zhang et al. (2017).

In this paper, we extend the study of eccentricity topological indices, based on the eccentricity, in chemical graph theory by involving an algebraic structures called a vector space. We consider a graph associated to a finite dimensional vector space over a finite field, and compute all the indices listed in the Table 1.

\section{Graph associated to a vector space}

Let $\mathbb{V}$ be a finite dimensional vector space over a field $\mathbb{F}$ with $\left\{\alpha_{1}, \alpha_{2}, \ldots, \alpha_{n}\right\}$ as a basis and $\theta$ as the null vector. Then any vector $v \in \mathbb{V}$ can be expressed uniquely as a linear combination of the form $v=a_{1} \alpha_{1}+a_{2} \alpha_{2}+\ldots+a_{n} \alpha_{n}$.
This representation of $v$ is its basic representation with respect to $\left\{\alpha_{1}, \alpha_{2}, \ldots, \alpha_{n}\right\}$. Now, the graph associated with $\mathbb{V}$, denoted by $\Gamma(\mathbb{V})$, is called a non-zero component graph, which is defined with respect to a basis $\left\{\alpha_{1}, \alpha_{2}, \ldots, \alpha_{n}\right\}$ as follows: the vertex set is $V(\Gamma(\mathbb{V}))=\mathbb{V}-\theta$; and two vertices $v_{1}, v_{2} \in V(\Gamma(\mathbb{V}))$ form an edge in $\Gamma(\mathbb{V})$ if $v_{1}$ and $v_{2}$ share at least one $\alpha_{i}$ with non-zero coefficient in their basic representation, i.e. their exists at least one $\alpha_{i}$ along which both $v_{1}$ and $v_{2}$ have non-zero components. Unless otherwise mention, we take the basis $\left\{\alpha_{1}, \alpha_{2}, \ldots, \alpha_{n}\right\}$ on which the graph is constructed (Das, 2016). Now, we state some basic results about $\Gamma(\mathbb{V})$, which will be useful in the sequel.

Theorem 1 (Das, 2016)

If $\mathbb{V}$ be an $n$-dimensional vector space over a field $\mathbb{F}$ with $q \geq 2$ elements, then the order of $\Gamma(\mathbb{V})$ is $q^{\mathrm{n}}-1$ and the size of $\Gamma(\mathbb{V})$ is $\underline{q^{2 n}-q^{n}+1-(2 q-1)^{n}}$.

\section{Theorem 2 (Das, 2016)}

Let $\mathbb{V}$ be a vector space over a finite field $\mathbb{F}$ with $q \geq 2$ elements and $\Gamma(\mathbb{V})$ be its associated graph with respect to basis $\left\{\alpha_{1}, \alpha_{2}, \ldots, \alpha_{n}\right\}$. Then, the degree of the vertex $c_{1} \alpha_{i_{1}}+c_{2} \alpha_{i_{2}}+\ldots+c_{n} \alpha_{i}$, where $c_{1}, c_{2}, \ldots, c_{k} \neq 0$, is $\left(q^{k}-1\right) q^{n-k}-1$

\section{Theorem 3 (Das, 2016)}

$\Gamma(\mathbb{V})$ is connected and $\operatorname{diam}(\Gamma(\mathbb{V}))=2$.

\section{Theorem 4 (Das, 2016)}

$\Gamma(\mathbb{V})$ is a complete graph if and only if $\mathbb{V}$ is 1 -dimensional.

Let $\mathbb{V}$ be an $n$-dimensional vector space, $n \geq 1$, over a field $\mathbb{F}$ of order $q \geq 2$. Then there are $\left(\begin{array}{l}n \\ k\end{array}\right)(q-1)^{k}$ unique vectors $\alpha_{1} v_{1}+\alpha_{2} v_{2}+\ldots+\alpha_{k} v_{k}$ of length $k$ in $\Gamma(\mathbb{V})$ for each $1 \leq k \leq n$. Let us denote the vertex corresponds to the $i$ th vector of length $k$ by $v_{i}^{k}$, where $1 \leq i \leq\left(\begin{array}{l}n \\ k\end{array}\right)(q-1)^{k}$ and $1 \leq k \leq n$. So the vertex set of $\Gamma(\mathbb{V})$ is $\left\{v_{i}^{k} ; 1 \leq k \leq n, 1 \leq i \leq\left(\begin{array}{l}n \\ k\end{array}\right)(q-1)^{k}\right\}$. Let us denote the degree of a vertex $v_{i}^{k}, 1 \leq i \leq\left(\begin{array}{l}n \\ k\end{array}\right)(q-1)^{k}$, by $d_{k}$, then $d_{k}=\left(q^{k}-1\right) q^{n-k}-1$ for $1 \leq k \leq n$.

For $n=1$ and $q \geq 2, \Gamma(\mathbb{V})$ is a complete graph by Theorem 4, so one can trivially find all the topological indices in this particular case. We consider $\Gamma(\mathbb{V})$ for more than 1-dimensional vector spaces in the context of previously defined eccentricity based topological indices. 
Table 1: Eccentricity based topological indices.

\begin{tabular}{|c|c|c|}
\hline Notation & Formula & Index name (reference) \\
\hline$\xi(G)$ & $\sum_{v \in V(G)} \operatorname{ecc}(v)$ & $\begin{array}{l}\text { Total eccentricity index } \\
\text { (Sharma et al., 1997) }\end{array}$ \\
\hline $\operatorname{avec}(G)$ & $\frac{\xi(G)}{|G|}$ & $\begin{array}{l}\text { Average eccentricity index } \\
\text { (Dankelmann et al., 2004) }\end{array}$ \\
\hline$\xi^{c}(G)$ & $\sum_{v \in V(G)} \operatorname{ecc}(v) d(v)$ & $\begin{array}{l}\text { Eccentric connectivity index } \\
\text { (Sharma et al., 1997) }\end{array}$ \\
\hline$\xi^{d}(G)$ & $\sum_{v \in V(G)} e c c(v) D(v \mid G)$ & $\begin{array}{l}\text { Eccentric distance sum index } \\
\text { (Gupta et al., 2002) }\end{array}$ \\
\hline$\xi^{s v}(G)$ & $\sum_{v \in V(G)} \frac{e c c(v) D(v \mid G)}{d(v)}$ & $\begin{array}{l}\text { Adjacent eccentric distance sum index } \\
\text { (Sardana and Madan, 2002) }\end{array}$ \\
\hline$\xi^{c e}(G)$ & $\sum_{v \in V(G)} \frac{d(v)}{\operatorname{ecc}(v)}$ & $\begin{array}{l}\text { Connective eccentricity index } \\
\text { (Yu and Feng, 2013) }\end{array}$ \\
\hline$G A_{4}(G)$ & $\sum_{u v \in E(G)} \frac{2 \sqrt{e c c}(u) \times e c c(v)}{e c c(u)+e c c(v)}$ & $\begin{array}{l}\text { Geometric arithmetic index } \\
\text { (Ghorbani and Khaki, 2010) }\end{array}$ \\
\hline$A B C_{5}(G)$ & $\sum_{u v \in E(G)} \frac{\sqrt{\operatorname{ecc}(u)+e c c(v)-2}}{\operatorname{ecc}(u) \times \operatorname{ecc}(v)}$ & $\begin{array}{l}\text { Atomic bond connectivity index } \\
\text { (Farahani, 2013) }\end{array}$ \\
\hline$M_{1}^{\star}(G)$ & $\sum_{u v \in E(G)}(e c c(u)+e c c(v))$ & $\begin{array}{l}\text { First Zagreb eccentricity index } \\
\text { (Ghorbani and Hosseioninzadeh, 2012; Vukiĉević and Graovac, 2010) }\end{array}$ \\
\hline$M_{1}^{\star \star}(G)$ & $\sum_{v \in V(G)}(e c c(v))^{2}$ & $\begin{array}{l}\text { Second Zagreb eccentricity index } \\
\text { (Ghorbani and Hosseioninzadeh, 2012; Vukiĉević and Graovac, 2010) }\end{array}$ \\
\hline$M_{2}^{*}(G)$ & $\sum_{u v \in E(G)} \operatorname{ecc}(u) e c c(v)$ & $\begin{array}{l}\text { Third Zagreb eccentricity index } \\
\text { (Ghorbani and Hosseioninzadeh, 2012; Vukiĉević and Graovac, 2010) }\end{array}$ \\
\hline
\end{tabular}

\section{Methodology}

Some graph theoretical parameters such as path, distance, eccentricity, diameter, degree, etc. along with vertex partitioning method are used to construct some useful tools for investigating our main results. We also use combinatorial computing and binomial expansion theorem to find the required indices. Moreover, we use maple software (Maplesoft, McKinney, TX, USA) (see: https://en.wikipedia.org/wiki/Maplesoftware) for plotting our mathematical results, mathematical calculations and verifications, and to provide a numeric comparison of the investigated indices.

\section{Construction}

In this section, we construct/illustrate some useful results to compute the required indices. Let us denote the dimension of a vector space $\mathbb{V}$ with $\operatorname{dim}(\mathbb{V})$, and the order of a field $\mathbb{F}$ with $o(\mathbb{F})$.

\section{Remark 5}

If $\operatorname{dim}(\mathbb{V})=n \geq 2$ and $o(\mathbb{F})=q \geq 2$, then for all $1 \leq k \leq n$ and for each $1 \leq i \leq\left(\begin{array}{l}n \\ k\end{array}\right)(q-1)^{k}$, note that $d\left(v_{i}^{k}, v\right)=1$ or 2 , by Theorem 3 , for any $v \in V-\left\{v_{i}^{k}\right\}$. Since the number of vertices 
lying at distance 1 from $v_{i}^{k}$ in $\Gamma(\mathbb{V})$ is the degree $d_{k}$ of $v_{i}^{k}$, so the number of vertices lying at distance 2 from $v_{i}^{k}$ is

$$
|\Gamma(\mathbb{V})|-d_{k}-1=\left(q^{n}-1\right)-\left(q^{n-k}\left(q^{k}-1\right)-1\right)-1=q^{n-k}-1 .
$$

\section{Remark 6}

As the diameter of $\Gamma(\mathbb{V})$ is 2, by Theorem 3, therefore, $\operatorname{ecc}\left(v_{1}^{n}\right)=1$ for all $1 \leq i \leq(q-1)^{n}$, and $\operatorname{ecc}\left(v_{i}^{k}\right)=2$ for all $1 \leq i \leq\left(\begin{array}{l}n \\ k\end{array}\right)(q-1)^{k}$ and for each $1 \leq k \leq n-1$.

The following proposition provides the distance number of each vertex of $\Gamma(\mathbb{V})$.

\section{Proposition 7}

Let $\operatorname{dim}(\mathbb{V})=n \geq 2$ and $o(\mathbb{F})=q \geq 2$, then for any $1 \leq k \leq n$ and for each $1 \leq i \leq\left(\begin{array}{l}n \\ k\end{array}\right)(q-1)^{k}$, we have $D\left(v_{i}^{k} \mid \Gamma(\mathbb{V})\right)=q^{n-k}\left(q^{k}+1\right)-3$.

\section{Proof}

Using Remark 5, the distance number of any vertex of $\Gamma(\mathbb{V})$ is:

$D\left(v_{i}^{k} \mid \Gamma(\mathbb{V})\right)=\left[q^{n-k}\left(q^{k}-1\right)-1\right](1)+\left[q^{n-k}-1\right](2)=q^{n-k}\left(q^{k}+1\right)-3$.

The notation $a \sim b$ is a type of an edge whose end vertices have degrees $a$ and $b$. The following result gives edge partition of $\Gamma(\mathbb{V})$ according to the eccentricity of each vertex.

\section{Proposition 8}

Let $\operatorname{dim}(\mathbb{V})=n \geq 2$ and $o(\mathbb{F})=q \geq 2$, then the edge partition of $\Gamma(\mathbb{V})$ according to the eccentricity of each vertex is:

(1) $\left(\begin{array}{c}(q-1)^{n} \\ 2\end{array}\right)$ edges of type $1 \sim 1$,

(2) $(q-1)^{n}\left(q^{n}-(q-1)^{n}+1\right)$ edges of type $1 \sim 2$,

(3) $\left.\frac{1}{2}\left[q^{2 n}-q^{n}-(2 q-1)^{n}-(q-1)^{n}\left(2 q^{n}-(q-1)^{n}\right)+1\right)+1\right]$

edges of type $2 \sim 2$.

where $a \sim b$ denotes an edge whose one end vertex has the eccentricity $a$ and the other end vertex has the eccentricity $b$.

\section{Proof}

By Remark 6, there are three types of edges of $\Gamma(\mathbb{V})$ : $1 \sim 1 ; 1 \sim 2$ and $2 \sim 2$. The number of edges in each type can be found as follows:

(1) Edges of type 1 1: As $\operatorname{ecc}\left(v_{i}^{n}\right)=1$ for all $1 \leq i \leq(q-1)^{n}$ and the subgraph of $\Gamma(\mathbb{V})$ induced by the vertices $v_{i}^{n}$ for all $1 \leq i \leq(q-1)^{n}$ is a complete graph $K_{(q-1)^{n}}$, so the number of edges of type $1 \sim 1$ is the size of $K_{(q-1)^{n}}^{(q-1)^{n}}$, which is $\left(\begin{array}{c}(q-1)^{n} \\ 2\end{array}\right)$.

(2) Edges of type 1 2: In $\Gamma(\mathbb{V})$, two types of edges, $1 \sim 1$ and $1 \sim 2$, are counted to form a degree $d_{n}$ of a vertex $v_{i}^{n}$, $1 \leq i \leq(q-1)^{n}$. Thus, the number of edges of type $1 \sim 2$ is obtained from the degree $d_{n}$ of $v_{i}^{n}$ by subtracting the number of edges of type $1 \sim 1$. Note that, $(q-1)^{n}-1$ edges of type $1 \sim 1$ are counted to form a degree $d_{n}$, because every two vertices having degree $d_{n}$ are adjacent in $\Gamma(\mathbb{V})$. It follows that $d_{n}-(q-1)^{n}+1$ edges of type $1 \sim 2$ are counted to form each degree $d_{n}$. Since there are $(q-1)^{n}$ vertices having degree $d_{n}$, so the number of edges of type $1 \sim 2$ is

$$
(q-1)^{n}\left(d_{n}-(q-1)^{n}+1\right)=(q-1)^{n}\left(q^{n}-(q-1)^{n}+1\right) .
$$

(3) Edges of type 2 2: The number of edges of type $2 \sim 2$ can be obtained by the formula Size of $\Gamma(\mathbb{V})$ - the number of edges of type 1 1-the number of edges of type $2 \sim 2$, which yields that

$$
\begin{aligned}
& \frac{1}{2}\left(q^{2 n}-q^{n}-(2 q-1)^{n}+1\right)-\frac{1}{2}(q-1)^{n}\left((q-1)^{n}-1\right) \\
& \quad-(q-1)^{n}\left(q^{n}-(q-1)^{n}+1\right) \\
& =\frac{1}{2}\left(q^{2 n}-q^{n}-(2 q-1)^{n}-(q-1)^{n}\left(2 q^{n}-\left(q-1^{n}+1\right) .\right.\right.
\end{aligned}
$$

\section{Results}

The total eccentricity index of $\Gamma(\mathbb{V})$ is computed in the following result:

\section{Theorem 9}

Let $G=\Gamma(\mathbb{V})$ be a non-zero component graph of a vector space $\mathbb{V}$ of dimension $n \geq 2$ over a finite field $\mathbb{F}$ of order $q \geq 2$. Then $\xi(G)=2\left(q^{n}-1\right)-(q-1)^{n}$.

\section{Proof}

Using Remark 6 in the formula of total eccentricity index, we have 


$$
\begin{aligned}
\xi(G) & =\sum_{k=1}^{n} \sum_{i=1}^{\left(\begin{array}{c}
n \\
k
\end{array}\right)(q-1)^{k}} \operatorname{ecc}\left(v_{i}^{k}\right) \\
& =\left[\left(q^{n}-1\right)-(q-1)^{n}\right](2)+(q-1)^{n}=2\left(q^{n}-1\right)-(q-1)^{n} .
\end{aligned}
$$

Using the total eccentricity index, computed in Theorem 9, in the formula of the average eccentricity index, we get the following result:

\section{Theorem 10}

Let $G=\Gamma(\mathbb{V})$ be a non-zero component graph of a vector space $\mathbb{V}$ of dimension $n \geq 2$ over a finite field $\mathbb{F}$ of order $q \geq 2$. Then $\operatorname{avec}(G)=2-\frac{(q-1)^{n}}{q^{n}-1}$.

In the following result, we compute the connective eccentricity index of $\Gamma(\mathbb{V})$

\section{Theorem 11}

Let $G=\Gamma(\mathbb{V})$ be a non-zero component graph of a vector space $\mathbb{V}$ of dimension $n \geq 2$ over a finite field $\mathbb{F}$ of order $q \geq 2$. Then

$$
\xi^{c e}(G)=\frac{1}{2}\left[q^{n}\left(q^{n}-1\right)-(2 q-1)^{n}+(q-1)^{n}\left(q^{n}-2\right)+1\right] .
$$

\section{Proof}

Using the degree and eccentricity of each vertex, given in Remark 6, in the formula of connective eccentricity index, we have

$$
\begin{aligned}
& \xi^{c e}(G)=\sum_{k=1} \sum_{i=1} \frac{d\left(\begin{array}{l}
n \\
k
\end{array}\right)(q-1)^{k}}{e c c\left(v_{i}^{k}\right)} \\
&=\frac{\left(\begin{array}{c}
n \\
1
\end{array}\right)(q-1)^{1}\left(\left(q^{1}-1\right) q^{n-1}-1\right)}{2} \\
&+\left(\begin{array}{c}
n \\
2
\end{array}\right)(q-1)^{2}\left(\left(q^{2}-1\right) q^{n-2}-1\right) \\
& 2
\end{aligned}
$$

$$
\begin{aligned}
& +\frac{\left(\begin{array}{c}
n \\
n-1
\end{array}\right)(q-1)^{n-1}\left(\left(q^{n-1}-1\right) q^{n-(n-1)}-1\right)}{2} \\
& +\frac{\left(\begin{array}{c}
n \\
n
\end{array}\right)(q-1)^{n}\left(\left(q^{n}-1\right) q^{n-n}-1\right)}{1} \\
& =\frac{1}{2}\left(\begin{array}{c}
n \\
1
\end{array}\right)\left[(q-1)^{1} q^{n}-(q-1)^{1} q^{n-1}-(q-1)^{1}\right] \\
& +\frac{1}{2}\left(\begin{array}{c}
n \\
2
\end{array}\right)\left[(q-1)^{2} q^{n}-(q-1)^{2} q^{n-2}-(q-1)^{2}\right]+\ldots \\
& +\frac{1}{2}\left(\begin{array}{c}
n \\
n
\end{array}\right)\left[(q-1)^{n} q^{n}-(q-1)^{n} q^{n-1}-(q-1)^{n}\right] \\
& +\frac{(q-1)^{n}\left(q^{n}-2\right)}{2} .
\end{aligned}
$$

Few more simplifications, using binomial expansions, provide the required results.

The eccentric connectivity index of $\Gamma(\mathbb{V})$ is computed in the next result.

\section{Theorem 12}

Let $G=\Gamma(\mathbb{V})$ be a non-zero component graph of a vector space $\mathbb{V}$ of dimension $n \geq 2$ over a finite field $\mathbb{F}$ of order $q \geq 2$. Then

$$
\xi^{c}(G)=2\left(q^{2 n}-q^{n}+1-(2 q-1)^{n}\right)+(q-1)^{n} .
$$

\section{Proof}

Using the degree and eccentricity of each vertex, given in Remark 6, in the formula of eccentric connectivity index, we have

$$
\begin{aligned}
\xi^{c}(G) & =\sum_{k=1} \sum_{i=1} \operatorname{ecc}\left(v_{i}^{k}\right) d\left(v_{i}^{k}\right) \\
& =\left(\begin{array}{c}
n \\
1
\end{array}\right)(q-1)^{k} \\
& +\left(q^{n}-q^{n-1}-1\right)(2) \\
& +\left(\begin{array}{c}
n \\
2
\end{array}\right)(q-1)^{2}\left(q^{n}-q^{n-2}-1\right)(2)+\ldots
\end{aligned}
$$




$$
\begin{aligned}
& +\left(\begin{array}{c}
n \\
n-1
\end{array}\right)(q-1)^{n-1}\left(q^{n}-q^{1}-1\right)(2) \\
& +\left(\begin{array}{l}
n \\
n
\end{array}\right)(q-1)^{n}\left(q^{n}-q^{n-n}-1\right)(1) \\
& =2\left(\begin{array}{c}
n \\
1
\end{array}\right)\left[(q-1)^{1} q^{n}+(q-1)^{1} q^{n-1}-(q-1)^{1}\right] \\
& +2\left(\begin{array}{c}
n \\
2
\end{array}\right)\left[(q-1)^{2} q^{n}-(q-1)^{2} q^{n-2}-(q-1)^{2}\right]+\ldots \\
& +2\left(\begin{array}{l}
n \\
n
\end{array}\right)\left[(q-1)^{n} q^{n}-(q-1)^{n} q^{n-n}-(q-1)^{n}\right] \\
& -\left(\begin{array}{l}
n \\
n
\end{array}\right)\left[(q-1)^{n} q^{n}-(q-1)^{n} q^{n-n}-(q-1)^{n}\right] \\
& =q^{n}\left[\begin{array}{l}
2\left(-1+1+\left(\begin{array}{c}
n \\
1
\end{array}\right)(q-1)+\left(\begin{array}{c}
n \\
2
\end{array}\right)(q-1)^{2}+\ldots+\left(\begin{array}{l}
n \\
n
\end{array}\right)(q-1)^{n}\right) \\
-\left(\begin{array}{l}
n \\
n
\end{array}\right)(q-1)^{n}
\end{array}\right] \\
& -\left[\begin{array}{l}
2\left(-q^{n}+q^{n}+\left(\begin{array}{c}
n \\
1
\end{array}\right)(q-1)^{1} q^{n-1}+\ldots+\left(\begin{array}{l}
n \\
n
\end{array}\right)(q-1)^{n} q^{n-n}\right) \\
-\left(\begin{array}{c}
n \\
n
\end{array}\right)(q-1)^{n} q^{n-n}
\end{array}\right] \\
& -\left[2\left(-1+1+\left(\begin{array}{c}
n \\
1
\end{array}\right)(q-1)+\ldots+\left(\begin{array}{l}
n \\
n
\end{array}\right)(q-1)^{n}\right)-\left(\begin{array}{l}
n \\
n
\end{array}\right)(q-1)^{n}\right] .
\end{aligned}
$$

After some simple calculations, using binomial expansions, we get the required index.

The investigation of the eccentric distance sum index of $\Gamma(\mathbb{V})$ is given in the following result:

\section{Theorem 13}

Let $G=\Gamma(\mathbb{V})$ be a non-zero component graph of a vector space $\mathbb{V}$ of dimension $n \geq 2$ over a finite field $\mathbb{F}$ of order $q \geq 2$. Then

$$
\xi^{d}(G)=2\left[(2 q-1)^{n}+q^{2 n}-4 q^{n}+3\right]-\left[(q-1)^{n}\left(q^{n}-2\right)\right] .
$$

\section{Proof}

Using the eccentricity of each vertex, given in Remark 6 , and the distance number of each vertex, given in
Proposition 7, in the formula of eccentric distance sum index, we have

$$
\begin{aligned}
& n\left(\begin{array}{l}
n \\
k
\end{array}\right)(q-1)^{k} \\
& \xi^{d}(G)=\sum_{k=1} \sum_{i=1} \operatorname{ecc}\left(v_{i}^{k}\right) D\left(v_{i}^{k} \mid G\right) \\
& =2\left(\begin{array}{c}
n \\
1
\end{array}\right)(q-1)^{1}\left(q^{n-1}\left(q^{1}+1\right)-3\right) \\
& +2\left(\begin{array}{c}
n \\
2
\end{array}\right)(q-1)^{2}\left(q^{n-2}\left(q^{2}+1\right)-3\right)+\ldots \\
& +2\left(\begin{array}{c}
n \\
n-1
\end{array}\right)(q-1)^{n-1}\left(q^{n-(n-1)}\left(q^{n-1}+1\right)-3\right) \\
& +\left(\begin{array}{l}
n \\
n
\end{array}\right)(q-1)^{n}\left(q^{n-n}\left(q^{n}+1\right)-3\right) \\
& =2\left[\begin{array}{l}
\left(\begin{array}{c}
n \\
1
\end{array}\right)(q-1)^{1}\left(q^{n}+q^{n-1}-3\right)+\ldots+\left(\begin{array}{c}
n \\
n-1
\end{array}\right)(q-1)^{n-1} \\
\left(q^{n}+q^{n-(n-1)}-3\right)
\end{array}\right] \\
& -\left(\begin{array}{l}
n \\
n
\end{array}\right)(q-1)^{n}\left(q^{n}+q^{n-n}-3\right) \\
& =2 q^{n}\left[-1+1+\left(\begin{array}{c}
n \\
1
\end{array}\right)(q-1)+\left(\begin{array}{c}
n \\
2
\end{array}\right)(q-1)^{2}+\ldots+\left(\begin{array}{l}
n \\
n
\end{array}\right)(q-1)^{n}\right] \\
& +2\left[-q^{n}+q^{n}+\left(\begin{array}{c}
n \\
1
\end{array}\right)(q-1) q^{n-1}++\ldots+\left(\begin{array}{l}
n \\
n
\end{array}\right)(q-1)^{n} q^{n-n}\right] \\
& -6\left[-1+1+\left(\begin{array}{c}
n \\
1
\end{array}\right)(q-1)+\left(\begin{array}{c}
n \\
2
\end{array}\right)(q-1)^{2}+\ldots+\left(\begin{array}{l}
n \\
n
\end{array}\right)(q-1)^{n}\right] \\
& -\left((q-1)^{n}\left(q^{n}-2\right)\right) \text {. }
\end{aligned}
$$

Using binomial expansions and by performing some simplifications, we get the required index.

The adjacent eccentric distance sum index of $\Gamma(\mathbb{V})$ is investigated in the following result:

\section{Theorem 14}

Let $G=\Gamma(\mathbb{V})$ be a non-zero component graph of a vector space $\mathbb{V}$ of dimension $n \geq 2$ over a finite field $\mathbb{F}$ of order $q \geq 2$.

Then

$$
\xi^{s v}(G)=(q-1)^{n}+2 \sum_{j=1}^{n-1}\left(\begin{array}{c}
n \\
j
\end{array}\right)(q-1)^{j}\left(\frac{q^{n}+q^{n-j}-3}{q^{n}-q^{n-j}-1}\right) .
$$




\section{Proof}

Using the degree, eccentricity and distance number of each vertex, given in Remark 6 and Proposition 7, in the formula of adjacent eccentric distance sum index, we have

$$
\begin{aligned}
\xi^{d}(G) & =\sum_{k=1} \sum_{i=1} \frac{\operatorname{ecc}\left(v_{i}^{n}\right) D\left(v_{i}^{k} \mid G\right)}{d\left(v_{i}^{k}\right)} \\
& =\sum_{k=1}^{n}\left(\begin{array}{l}
n \\
k
\end{array}\right)(q-1)^{k} \frac{\operatorname{ecc}\left(v_{i}^{k}\right)\left(q^{n-k}\left(q^{k}+1\right)-3\right)}{\left(\left(q^{k}-1\right) q^{n-k}-1\right)}
\end{aligned}
$$$$
=\left(\begin{array}{c}
n \\
1
\end{array}\right)(q-1)^{1} \frac{\operatorname{ecc}\left(v_{i}^{1}\right)\left(q^{n-1}\left(q^{1}+1\right)-3\right)}{\left(\left(q^{1}-1\right) q^{n-1}-1\right)}
$$$$
+\left(\begin{array}{l}
n \\
2
\end{array}\right)(q-1)^{2} \frac{\operatorname{ecc}\left(v_{i}^{2}\right)\left(q^{n-2}\left(q^{2}+1\right)-3\right)}{\left(\left(q^{2}-1\right) q^{n-2}-1\right)}
$$$$
+\ldots+\left(\begin{array}{c}
n \\
n-1
\end{array}\right)(q-1)^{n-1} \frac{\operatorname{ecc}\left(v_{i}^{n-1}\right)\left(q^{n-(n-1)}\left(q^{n-1}+1\right)-3\right)}{\left(\left(q^{n-1}-1\right) q^{n-(n-1)}-1\right)}
$$$$
+\left(\begin{array}{c}
n \\
n
\end{array}\right)(q-1)^{n} \frac{\operatorname{ecc}\left(v_{i}^{n}\right)\left(q^{n-n}\left(q^{n}+1\right)-3\right)}{\left(\left(q^{n}-1\right) q^{n-n}-1\right)}
$$$$
=\left(\begin{array}{c}
n \\
1
\end{array}\right)(q-1)^{1} \frac{2\left(q^{n-1}\left(q^{1}+1\right)-3\right)}{\left(\left(q^{1}-1\right) q^{n-1}-1\right)}
$$$$
+\left(\begin{array}{c}
n \\
2
\end{array}\right)(q-1)^{2} \frac{2\left(q^{n-2}\left(q^{2}+1\right)-3\right)}{\left(\left(q^{2}-1\right) q^{n-2}-1\right)}+\ldots
$$$$
+\left(\begin{array}{c}
n \\
n-1
\end{array}\right)(q-1)^{n-1} \frac{2\left(q^{n-(n-1)}\left(q^{n-1}+1\right)-3\right)}{\left(\left(q^{n-1}-1\right) q^{n-(n-1)}-1\right)}
$$$$
+\left(\begin{array}{l}
n \\
n
\end{array}\right)(q-1)^{n} \frac{\left(q^{n-n}\left(q^{n}+1\right)-3\right)}{\left(\left(q^{n}-1\right) q^{n-n}-1\right)}
$$

$$
=2 \sum_{j=1}^{n-1}\left(\begin{array}{c}
n \\
j
\end{array}\right)(q-1)^{j}\left(\frac{q^{n}+q^{n-j}-3}{q^{n}-q^{n-j}-1}\right)+(q-1)^{n} .
$$

We get the required result after performing some calculations using binomial expansion.

The eccentricity based geometric arithmetic index of $\Gamma(\mathbb{V})$ is computed in the next result.

\section{Theorem 15}

Let $G=\Gamma(\mathbb{V})$ be a non-zero component graph of a vector space $\mathbb{V}$ of dimension $n \geq 2$ over a finite field $\mathbb{F}$ of order $q \geq 2$. Then

$$
\begin{aligned}
G A_{4}(G) & =\left(\frac{3-2 \sqrt{2}}{3}\right)(q-1)^{2 n} \\
& +\frac{1}{6}\left[\left[(4 \sqrt{2}-6)\left(q^{n}+1\right)\right](q-1)^{n}+3 q^{2 n}-3 q^{n}-3(2 q-1)^{n}+3\right] .
\end{aligned}
$$

\section{Proof}

Using the edge partition, given in Proposition 8, in the formula of geometric arithmetic index, we have

$$
\begin{aligned}
& G A_{4}(G)=\sum_{u \sim v} \frac{2 \sqrt{\operatorname{ecc}(u) \times e c c}(v)}{\operatorname{ecc}(u)+e c c(v)} \\
& =\left(\begin{array}{c}
(q-1)^{n} \\
2
\end{array}\right) \frac{2 \sqrt{1 \times 1}}{1+1}+\left[(q-1)^{n}\left(q^{n}-(q-1)^{n}+1\right)\right] \frac{2 \sqrt{1 \times 2}}{1+2} \\
& +\frac{1}{2}\left[q^{2 n}-q^{n}-(2 q-1)^{n}-(q-1)^{n}\left(2 q^{n}-(q-1)^{n}+1\right)+1\right] \frac{2 \sqrt{2 \times 2}}{2+2} \\
& =\frac{(q-1)^{n}\left((q-1)^{n}-1\right)}{2}+\left[(q-1)^{n}\left(q^{n}-(q-1)^{n}+1\right)\right] \frac{2 \sqrt{2}}{3} \\
& +\frac{1}{2}\left[q^{2 n}-q^{n}-(2 q-1)^{n}-(q-1)^{n}\left(2 q^{n}-(q-1)^{n}+1\right)+1\right] \\
& =\frac{1}{6}\left[(q-1)^{n}\left[\begin{array}{l}
3(q-1)^{n}-3+\left(4 q^{n}-4(q-1)^{n}+4\right) \sqrt{2}-6 q^{n} \\
+3(q-1)^{n}-3
\end{array}\right]\right]
\end{aligned}
$$




$$
\begin{aligned}
& +\frac{1}{6}\left[3 q^{2 n}-3 q^{n}-3(2 q-1)^{n}+3\right] \\
& =\frac{1}{6}\left[(q-1)^{n}\left[(6-4 \sqrt{2})(q-1)^{n}+(4 \sqrt{2}-6) q^{n}+4 \sqrt{2}-6\right]\right] \\
& +\frac{1}{6}\left[3 q^{2 n}-3 q^{n}-3(2 q-1)^{n}+3\right] .
\end{aligned}
$$

By performing some calculations, using binomial expansion, we get the required index.

The following result provides the formula for the eccentricity based $A B C$ index of $\Gamma(\mathbb{V})$.

\section{Theorem 16}

Let $G=\Gamma(\mathbb{V})$ be a non-zero component graph of a vector space $\mathbb{V}$ of dimension $n \geq 2$ over a finite field $\mathbb{F}$ of order $q \geq 2$. Then

$$
A B C_{5}(G)=\frac{1}{2 \sqrt{2}}\left[(q-1)^{n}\left(1-(q-1)^{n}\right)+q^{n}\left(q^{n}-1\right)-(2 q-1)^{n}+1\right] .
$$

\section{Proof}

Using the edge partition, given in Proposition 8, in the formula of $A B C$ index, we have

$$
\begin{aligned}
& A B C_{5}(G)=\sum_{u \sim v} \sqrt{\frac{e c c(u)+e c c(v)-2}{\operatorname{ecc}(u) \times e c c(v)}} \\
& =\left(\begin{array}{c}
(q-1)^{n} \\
2
\end{array}\right) \sqrt{\frac{1+1-2}{1 \times 1}}+\left[(q-1)^{n}\left(q^{n}-(q-1)^{n}+1\right)\right] \sqrt{\frac{1+2-2}{1 \times 2}} \\
& +\frac{1}{2}\left[q^{2 n}-q^{n}-(2 q-1)^{n}-(q-1)^{n}\left(2 q^{n}-(q-1)^{n}+1\right)+1\right] \sqrt{\frac{2+2-2}{2 \times 2}} \\
& =\frac{(q-1)^{n}\left((q-1)^{n}-1\right)}{2}(0)+\left[(q-1)^{n}\left(q^{n}-(q-1)^{n}+1\right)\right] \frac{1}{\sqrt{2}} \\
& +\frac{1}{2}\left[q^{2 n}-q^{n}-(2 q-1)^{n}-(q-1)^{n}\left(2 q^{n}-(q-1)^{n}+1\right)+1\right] \frac{1}{\sqrt{2}} \\
& =\frac{1}{2 \sqrt{2}}\left[2(q-1)^{n}\left(q^{n}-(q-1)^{n}+1\right)+q^{2 n}-q^{n}-(2 q-1)^{n}\right]
\end{aligned}
$$

$$
\begin{aligned}
& -\frac{1}{2 \sqrt{2}}\left[(q-1)^{n}\left(2 q^{n}-(q-1)^{n}+1\right)-1\right] \\
& =\frac{1}{2 \sqrt{2}}\left[(q-1)^{n}\left[2 q^{n}-2(q-1)^{n}+2-2 q^{n}+(q-1)^{n}-1\right]\right] \\
& +\left[q^{2 n}-q^{n}-(2 q-1)^{n}+1\right] \\
& =\frac{1}{2 \sqrt{2}}\left[(q-1)^{n}\left(1-(q-1)^{n}\right)+q^{n}\left(q^{n}-1\right)-(2 q-1)^{n}+1\right] .
\end{aligned}
$$

Hence, the required result is obtained.

The investigation of the first Zagreb eccentricity index of $\Gamma(\mathbb{V})$ is given in the following result:

\section{Theorem 17}

Let $G=\Gamma(\mathbb{V})$ be a non-zero component graph of a vector space $\mathbb{V}$ of dimension $n \geq 2$ over a finite field $\mathbb{F}$ of order $q \geq 2$. Then

$$
M_{1}^{\star}(G)=2 q^{n}\left(q^{n}-1\right)-q^{n}(q-1)^{n}-2(2 q-1)^{n}+2
$$

\section{Proof}

Using edge partition, given in Proposition 8, in the formula of the first Zagreb eccentricity index, we have

$$
\begin{aligned}
& M_{1}^{\star}(G)=\sum_{u \sim v}[\operatorname{ecc}(u)+e c c(v)] \\
&=\left(\begin{array}{c}
(q-1)^{n} \\
2
\end{array}\right)[1+1]+\left[(q-1)^{n}\left(q^{n}-(q-1)^{n}+1\right)\right][1+2] \\
&+\frac{1}{2}\left[q^{2 n}-q^{n}-(2 q-1)^{n}-(q-1)^{n}\left(2 q^{n}-(q-1)^{n}+1\right)+1\right][2+2] \\
&= \frac{(q-1)^{n}\left((q-1)^{n}-1\right)}{2}[2]+\left[(q-1)^{n}\left(q^{n}-(q-1)^{n}+1\right)\right][3] \\
&+\frac{1}{2}\left[q^{2 n}-q^{n}-(2 q-1)^{n}-(q-1)^{n}\left(2 q^{n}-(q-1)^{n}+1\right)+1\right][4] \\
&=(q-1)^{n}\left((q-1)^{n}-1\right)+3(q-1)^{n}\left(q^{n}-(q-1)^{n}+1\right)
\end{aligned}
$$




$$
\begin{aligned}
& -2\left[(q-1)^{n}\left(2 q^{n}-(q-1)^{n}\right)+1\right]+2\left(q^{2 n}-q^{n}-(2 q-1)^{n}+1\right) \\
& =(q-1)^{n}\left[(q-1)^{n}-1+3 q^{i n}-3(q-1)^{n}+3-4 q^{n}+2(q-1)^{n}-2\right] \\
& +2 q^{2 n}-2 q^{n}-2(2 q-1)^{n}+2 .
\end{aligned}
$$

Binomial expansion and some simplifications yield the required result.

The second Zagreb eccentricity index of $\Gamma(\mathbb{V})$ is computed in the next result.

\section{Theorem 18}

Let $G=\Gamma(\mathbb{V})$ be a non-zero component graph of a vector space $\mathbb{V}$ of dimension $n \geq 2$ over a finite field $\mathbb{F}$ of order $q \geq 2$. Then

$$
M_{1}^{\star \star}(G)=4 q^{n}-3(q-1)^{n}-4
$$

\section{Proof}

By using the eccentricity of each vertex, given in Remark 6 , in the formula of second Zagreb eccentricity index, we have

$$
\begin{aligned}
M_{1}^{* *}(G) & =\sum_{v_{i}^{k} \in V(G)}\left[\operatorname{ecc}\left(v_{i}^{k}\right)\right]^{2} \\
& =\left[\left(q^{n}-1\right)-(q-1)^{n}\right](2)^{2}+(q-1)^{n}(1)^{2} \\
& =4\left[\left(q^{n}-1\right)-(q-1)^{n}\right]+(q-1)^{n}(1) \\
& =4 q^{n}-4-4(q-1)^{n}+(q-1)^{n} \\
& =4 q^{n}-3(q-1)^{n}-4 .
\end{aligned}
$$

Hence, we get the required result.

The following result provides the formulation of the third Zagreb eccentricity index of $\Gamma(\mathbb{V})$.

\section{Theorem 19}

Let $G=\Gamma(\mathbb{V})$ be a non-zero component graph of a vector space $\mathbb{V}$ of dimension $n \geq 2$ over a finite field $\mathbb{F}$ of order $q \geq 2$. Then
$M_{2}^{\star}(G)=\frac{1}{2}(q-1)^{n}\left[(q-1)^{n}-4 q^{n}-1\right]+2 q^{n}\left(q^{n}-1\right)-2(2 q-1)^{n}+2$.

\section{Proof}

Using edge partition, given in Proposition 8, in the formula of the third Zagreb eccentricity index, we have

$$
\begin{aligned}
M_{2}^{*}(G) & =\sum_{u \sim v}[\operatorname{ecc}(u) \times e c c(v)] \\
& =(q-1)^{n}{ }_{2}(1 \times 1)+\left[(q-1)^{n}\left(q^{n}-(q-1)^{n}+1\right)\right](1 \times 2)
\end{aligned}
$$

$$
\begin{gathered}
+\frac{1}{2}\left[q^{2 n}-q^{n}-(2 q-1)^{n}-(q-1)^{n}\left(2 q^{n}-(q-1)^{n}+1\right)+1\right](2 \times 2) \\
=\frac{1}{2}(q-1)^{n}\left((q-1)^{n}-1\right)+(q-1)^{n}\left(2 q^{n}-2(q-1)^{n}+2\right)
\end{gathered}
$$

$$
\begin{aligned}
& +2 q^{2 n}-2 q^{n}-2(2 q-1)^{n}-(q-1)^{n}\left(4 q^{n}-2(q-1)^{n}+2\right)+2 \\
& =\frac{1}{2}(q-1)^{n}\left((q-1)^{n}-1\right)+(q-1)^{n}\left(2 q^{n}-2(q-1)^{n}+2\right) \\
& +2 q^{n}\left(q^{n}-1\right)-(q-1)^{n}\left(4 q^{n}-2(q-1)^{n}+2\right)+2-2(2 q-1)^{n} \\
& =\frac{1}{2}(q-1)^{n}\left[(q-1)^{n}-1+2\left(2 q^{n}-2(q-1)^{n}+2\right)-2\left(4 q^{n}-2(q-1)^{n}+2\right)\right] \\
& +2-2(2 q-1)^{n}+2 q^{n}\left(q^{n}-1\right) .
\end{aligned}
$$

Some easy simplifications with the help of binomial expansion give the required result.

\section{Comparisons and plots}

Using maple software (Maplesoft, McKinney, TX, USA), we provide a simple comparison of the investigated indices by plotting them and by constructing tables of their numeric values. We construct Tables 2 and 3 for different values of $q, n$, which depict that all the indices are in increasing order as the values of $q$ and $n$ are increasing, and their increasing behaviors are clearly shown in Figures 1-11 for certain values of $q$ and $n$. 
Table 2: Numeric comparison of the investigated indices at certain values of $q$ and $n$.

\begin{tabular}{|c|c|c|c|c|c|c|}
\hline$(q, n)$ & $\xi(\Gamma(\mathbb{V}))$ & $\operatorname{avec}(\Gamma(\mathbb{V}))$ & $\xi^{c e}(\Gamma(\mathbb{V}))$ & $\xi^{c}(\Gamma(\mathbb{V}))$ & $\xi^{d}(\Gamma(\mathbb{V}))$ & $\xi^{s v}(\Gamma(\mathbb{V}))$ \\
\hline$(2,2)$ & 5 & 1.7 & 3 & 9 & 22 & 13 \\
\hline$(2,3)$ & 13 & 1.86 & 18 & 61 & 118 & 27.4 \\
\hline$(2,4)$ & 29 & 1.933 & 87 & 321 & 538 & 52.78 \\
\hline$(2,5)$ & 61 & 1.9677 & 390 & 1501 & 2254 & 98.31 \\
\hline$(2,6)$ & 125 & 1.98413 & 1683 & 6609 & 9082 & 181.78 \\
\hline$(2,7)$ & 253 & 1.992126 & 7098 & 28141 & 35998 & 337.84 \\
\hline$(3,2)$ & 12 & 1.5 & 38 & 100 & 118 & 18.4 \\
\hline$(3,3)$ & 44 & 1.69 & 389 & 1164 & 1298 & 59.47 \\
\hline$(3,4)$ & 144 & 1.800 & 3560 & 11728 & 12466 & 173.84 \\
\hline$(3,5)$ & 452 & 1.8678 & 31697 & 111396 & 114698 & 504.89 \\
\hline$(3,6)$ & 1392 & 1.91209 & 280808 & 1030240 & 1041778 & 1482.16 \\
\hline$(3,7)$ & 4244 & 1.941446 & 2491169 & 9405444 & 9425018 & 4394.89 \\
\hline$(4,2)$ & 21 & 1.4 & 159 & 393 & 362 & 27.55 \\
\hline$(4,3)$ & 99 & 1.57 & 2682 & 7407 & 6698 & 115.98 \\
\hline$(4,4)$ & 429 & 1.682 & 41727 & 125841 & 113258 & 463.55 \\
\hline$(4,5)$ & 1803 & 1.7625 & 639546 & 2061735 & 1874234 & 1867.45 \\
\hline$(4,6)$ & 7461 & 1.82198 & 9819999 & 33311673 & 30772442 & 7576.76 \\
\hline$(4,7)$ & 30579 & 1.866508 & 151711482 & 535193247 & 502559498 & 30783.34 \\
\hline$(5,2)$ & 32 & 1.3 & 444 & 1056 & 850 & 38.74 \\
\hline$(5,3)$ & 184 & 1.48 & 11322 & 29608 & 23842 & 202.09 \\
\hline$(5,4)$ & 992 & 1.590 & 271464 & 767136 & 629890 & 102.91 \\
\hline$(5,5)$ & 5224 & 1.6722 & 6450702 & 19407928 & 16426402 & 5296.83 \\
\hline$(5,6)$ & 139864 & 1.73784 & 153792684 & 487191216 & 425227330 & 27286.82 \\
\hline$(5,7)$ & 156248 & 1.790282 & 3689310882 & 12197325448 & 10936004962 & 140109.41 \\
\hline$(6,2)$ & 45 & 1.29 & 995 & 2305 & 1702 & 51.90 \\
\hline$(6,3)$ & 305 & 1.419 & 35930 & 90345 & 67502 & 323.91 \\
\hline$(6,4)$ & 1965 & 1.5173 & 1236215 & 3327985 & 2569402 & 2005.38 \\
\hline$(6,5)$ & 12425 & 1.59807 & 42295550 & 120597825 & 96898502 & 12504.09 \\
\hline$(6,6)$ & 77685 & 1.665095 & 1451966435 & 4349943865 & 3627765802 & 77834.31 \\
\hline$(6,7)$ & 481745 & 1.7209174 & 50107120370 & 156688872105 & 134895219302 & 4820222.19 \\
\hline$(7,2)$ & 60 & 1.25 & 1938 & 4404 & 3062 & 67.02 \\
\hline$(7,3)$ & 468 & 1.368 & 94383 & 230436 & 163298 & 487.53 \\
\hline$(7,4)$ & 3504 & 1.4600 & 4421472 & 11468976 & 8458418 & 3546.27 \\
\hline$(7,5)$ & 25836 & 1.53731 & 206381415 & 564182076 & 434882954 & 25919.9 \\
\hline$(7,6)$ & 188640 & 1.603427 & 9662640588 & 27672732144 & 22202348402 & 188800.62 \\
\hline$(7,7)$ & 1367148 & 1.6600829 & 454349137083 & 1356319281516 & 1126026281018 & 1367450.35 \\
\hline
\end{tabular}

Table 3: Numeric comparison of the investigated indices at certain values of $q$ and $n$.

\begin{tabular}{|c|c|c|c|c|c|}
\hline$(q, n)$ & $G A_{4}(\Gamma(\mathbb{V}))$ & $A B C_{5}(\Gamma(\mathbb{V}))$ & $M_{1}^{*}(\Gamma(\mathbb{V}))$ & $M_{1}^{* *}(\Gamma(\mathbb{V}))$ & $M_{2}^{*}(\Gamma(\mathbb{V}))$ \\
\hline$(2,2)$ & 1.77 & 1.41 & 4 & 9 & 0 \\
\hline$(2,3)$ & 14.54 & 10.66 & 52 & 25 & 44 \\
\hline$(2,4)$ & 79.08 & 56.57 & 304 & 57 & 288 \\
\hline$(2,5)$ & 373.17 & 265.16 & 1468 & 121 & 1436 \\
\hline$(2,6)$ & 1648.34 & 1168.14 & 6544 & 249 & 6480 \\
\hline$(2,7)$ & 7027.68 & 4974.40 & 28012 & 505 & 27884 \\
\hline$(3,2)$ & 22.63 & 12.73 & 60 & 20 & 30 \\
\hline$(3,3)$ & 279.85 & 184.55 & 940 & 80 & 752 \\
\hline$(3,4)$ & 2867.61 & 1985.56 & 10416 & 272 & 9240 \\
\hline$(3,5)$ & 27543.02 & 19335.83 & 103588 & 872 & 96308 \\
\hline$(3,6)$ & 245106.29 & 180685.58 & 103588 & 2720 & 938880 \\
\hline$(3,7)$ & 2336248.89 & 1656893.32 & 9125380 & 8360 & 8853572 \\
\hline$(4,2)$ & 91.88 & 42.43 & 240 & 33 & 132 \\
\hline$(4,3)$ & 10504.95 & 1056.42 & 5652 & 171 & 4275 \\
\hline
\end{tabular}


Table 3: (Continued).

\begin{tabular}{|c|c|c|c|c|c|}
\hline$(q, n)$ & $G A_{4}(\Gamma(\mathbb{V}))$ & $A B C_{5}(\Gamma(\mathbb{V}))$ & $M_{1}^{*}(\Gamma(\mathbb{V}))$ & $M_{1}^{\star *}(\Gamma(\mathbb{V}))$ & $M_{2}^{*}(\Gamma(\mathbb{V}))$ \\
\hline$(4,4)$ & 30624.69 & 19940.41 & 105024 & 777 & 87528 \\
\hline$(4,5)$ & 504505.23 & 343632.68 & 1812660 & 3363 & 1593231 \\
\hline$(4,6)$ & 8187316.64 & 5700963.57 & 30324960 & 14193 & 27604332 \\
\hline$(4,7)$ & 132021927.06 & 92919045.25 & 499359252 & 58971 & 465917835 \\
\hline$(5,2)$ & 250.85 & 98.90 & 640 & 48 & 360 \\
\hline$(5,3)$ & 7159.07 & 3797.16 & 21544 & 304 & 15560 \\
\hline$(5,4)$ & 186302.87 & 112486.55 & 606880 & 1728 & 15560 \\
\hline$(5,5)$ & 4728625.44 & 3060322.79 & 16206904 & 9424 & 13530680 \\
\hline$(5,6)$ & 119095829.47 & 80193135.62 & 423187120 & 50208 & 13530680 \\
\hline$(5,7)$ & 2991473983.21 & 2991473983.21 & 10917309064 & 263344 & 9771518600 \\
\hline$(6,2)$ & 552.84 & 190.92 & 1380 & 65 & 780 \\
\hline$(6,3)$ & 21897.30 & 10468.72 & 63220 & 485 & 43970 \\
\hline$(6,4)$ & 807819.70 & 450313.88 & 2517360 & 3305 & 1902360 \\
\hline$(6,5)$ & 29317261.44 & 17866767.56 & 96294700 & 21725 & 76875950 \\
\hline$(6,6)$ & 1059751594.04 & 682654717.57 & 3620928240 & 139745 & 3013990740 \\
\hline$(6,7)$ & 38270493674.67 & 25541036167.4 & 134818793980 & 885365 & 116000512730 \\
\hline$(7,2)$ & 6063.18 & 326.68 & 2604 & 84 & 1470 \\
\hline$(7,3)$ & 55973.78 & 24278.51 & 156132 & 720 & 105264 \\
\hline$(7,4)$ & 2784943.85 & 1433842.85 & 8355984 & 5712 & 6083448 \\
\hline$(7,5)$ & 137026892.02 & 78357596.01 & 433483068 & 43896 & 333021036 \\
\hline$(7,6)$ & 6728737985.54 & 4122293610.46 & 22183653744 & 330624 & 17782989840 \\
\hline$(7,7)$ & 330376690613.97 & 212059773922.15 & 1125779668332 & 2454360 & 934422277164 \\
\hline
\end{tabular}

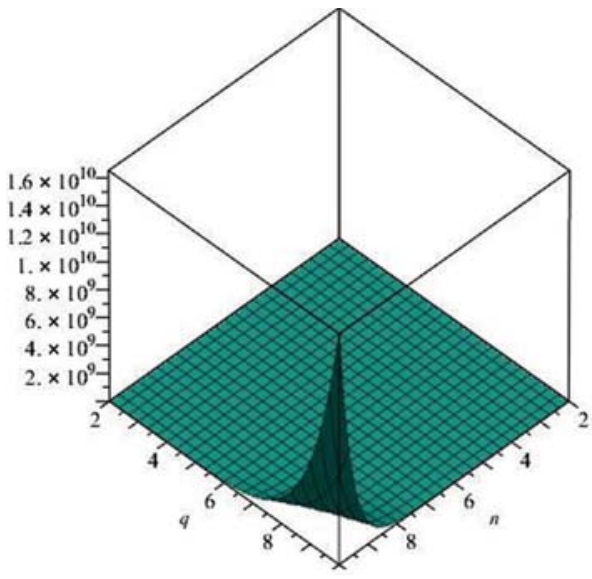

Figure 1: Plot of the total eccentricity.

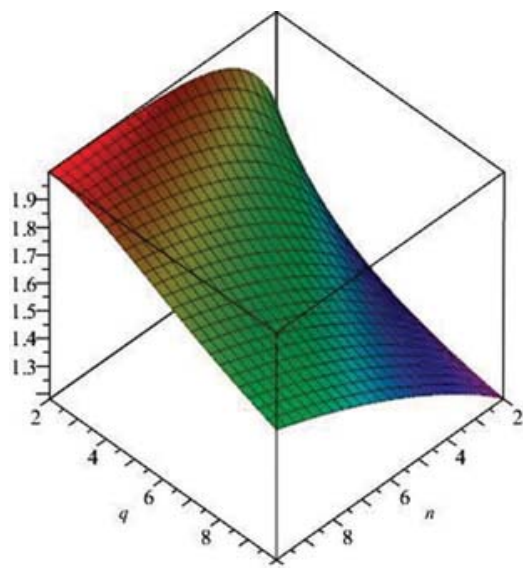

Figure 2: Plot of the average eccentricity.

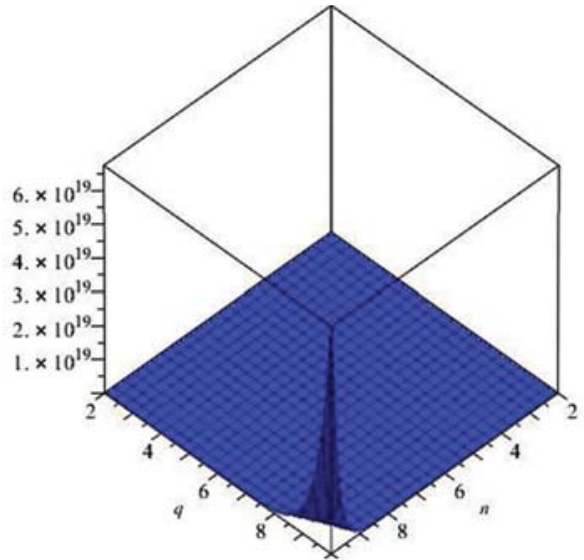

Figure 3: Plot of $\xi^{c e}$.

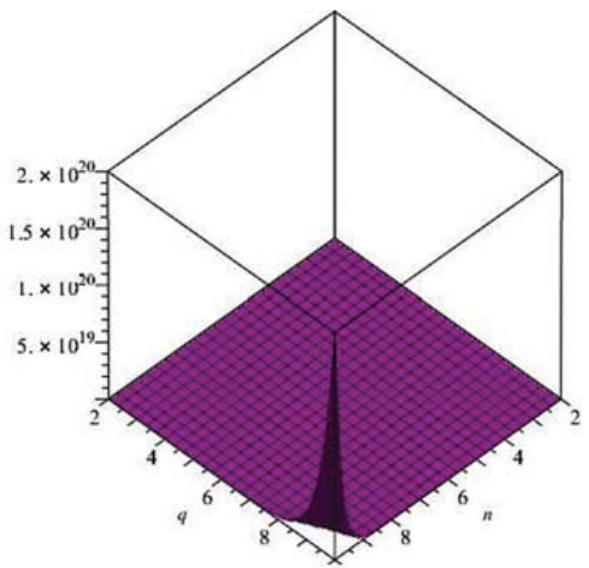

Figure 4: Plot of $\xi$. 


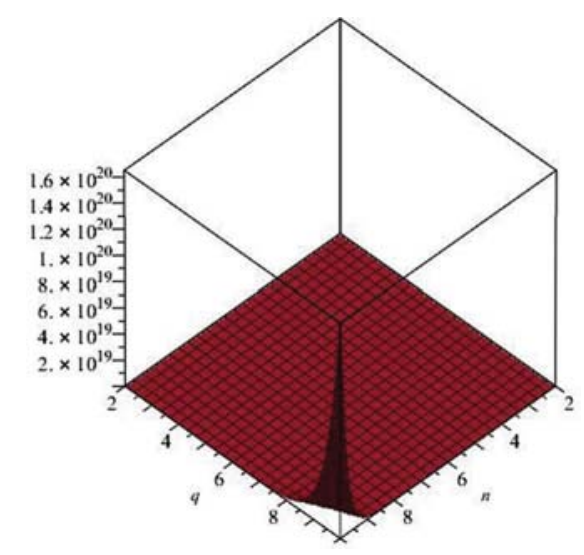

Figure 5: Plot of $\xi^{d}$.

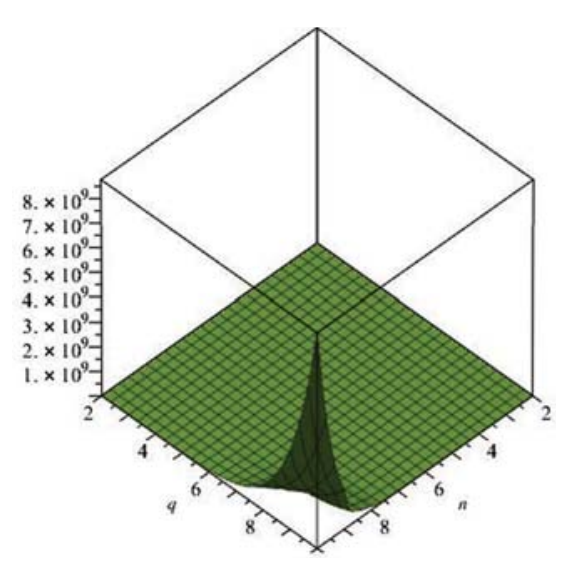

Figure 6: Plot of $\xi^{s v}$.

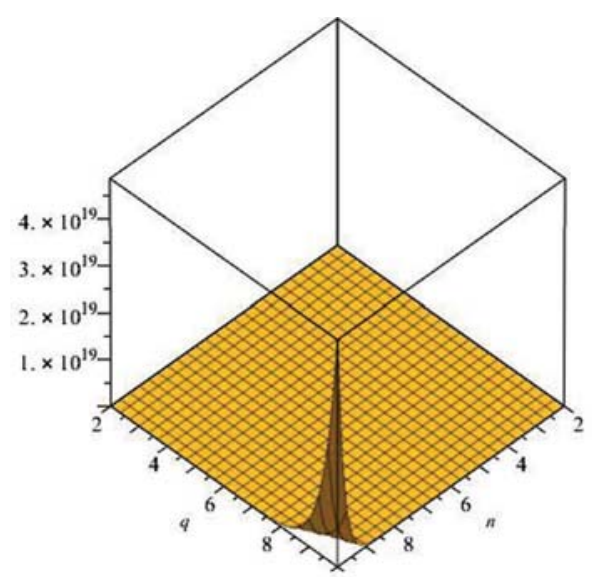

Figure 7: Plot of $G A_{4}$.

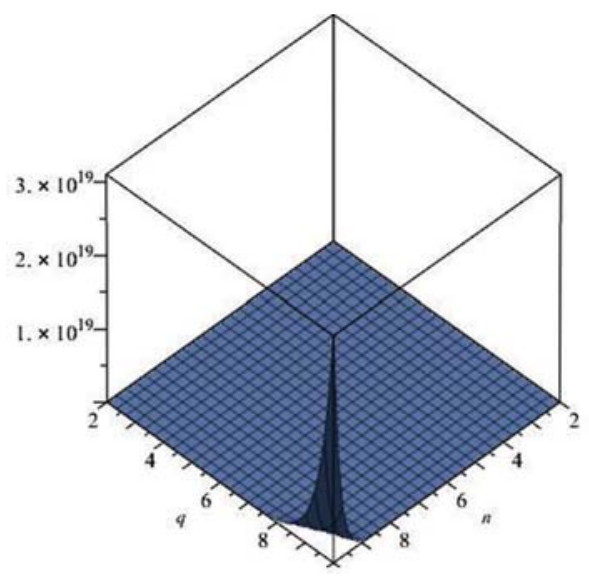

Figure 8: Plot of $A B C_{5}$.

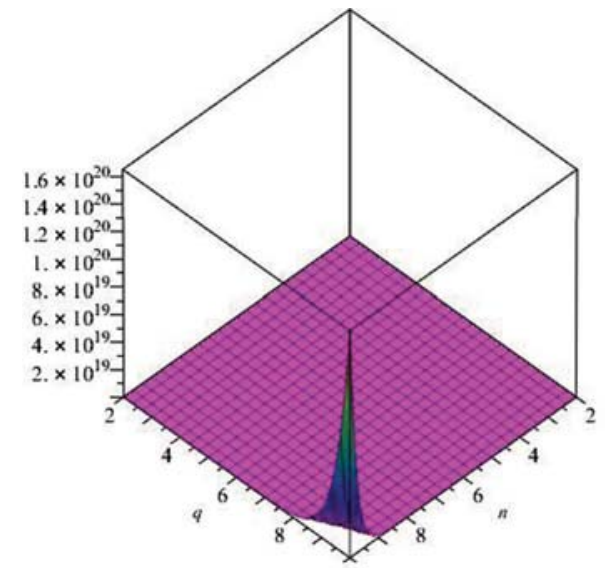

Figure 9: Plot of $M_{1}^{*}$.

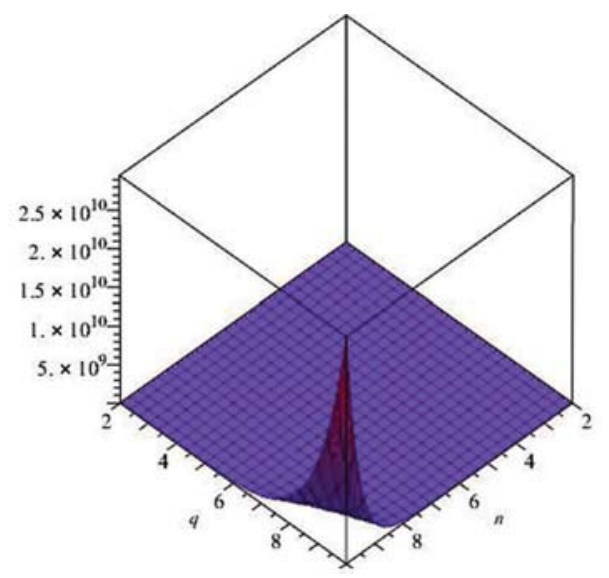

Figure 10: Plot of $M_{1}^{* *}$. 


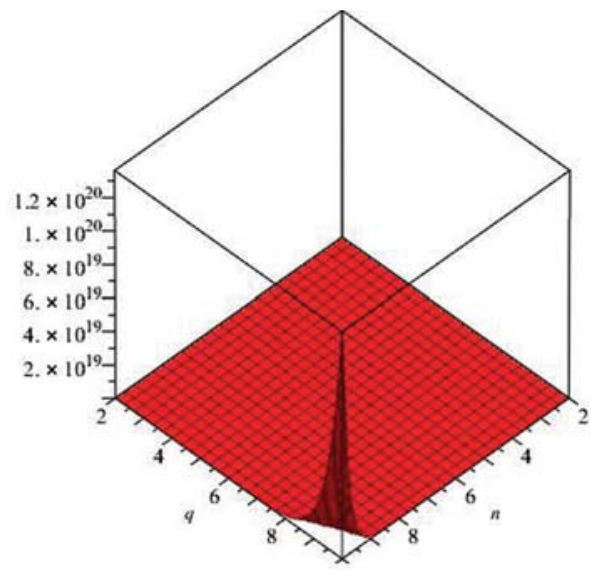

Figure 11: Plot of $M_{2}^{\star}$

Funding: This work was supported in part by Anhui Provincial Natural Science Foundation under Grant 2008085J01, the Humanities and Social Science Youth Foundation of the Ministry of Education of China under Grant 20YJC630029; the Natural Science Foundation of Anhui Province under Grant 2008085MG228 and 1908085MG228; the Scientific Research Starting Foundation of Anhui Jianzhu University under Grant 2019QDZ09 and 2019QDZ04.

\section{References}

Bruckler F.M., Došlíc T., Graovac A., Gutman I., On a class of distancebased molecular stucture descriptors. Chem. Phys. Lett., 2011, 503, 336-338.

Dankelmann P., Goddard W., Swart C.S., The average eccentricityof a graph and its subgraphs. Utilitas Mathematica, 2004, 65, 41-51.

Das A., Non-zero component graph of finite dimensional vetor spaces, Commun. Algebra, 2016, 44, 9, 3918-3926.

Deng H., Yang J., Xia F., A gernal modeling of some vertexdegree based topological indices in benzenoid systems and phenylenes. Comput. Math. Appl., 2011, 61, 3017-3023.

Farahani M.R., Eccentricity version of atom bond connectivity index of benzenoid family $A B C_{5}(\mathrm{HK})$. World Appl. Sci. J. Chem., 2013, 21, 1260-1265.

Gao W., Farahani M.R., Jamil M.K., The eccentricity version of atom-bond connectivity index of lineae polycene parallelogram benzenoid $A B C_{5} P(n, n)$. Acta Chim. Slov., 2016, 63, 376-379.

Ghorbani M., Hosseioninzadeh M.A., A new version of Zagreb indices. Fliomat, 2012, 6, 93-100.
Ghorbani M., Khaki A., A note on the forth version of geometricarithmetic index. Optoelectron. Adv. Mat., 2010, 4, 2212-2215.

Gupta S., Singh M., Madan A.K., Eccentric distance sum: A novel graph invariant for predicting biological and physical properties. J. Math. Anal. Appl., 2002, 275, 386-401.

Hinz A.M., Parisse D., The average eccentricity of Sierpinski graphs. Graph. Combinator., 2012, 5, 671-686.

Ilic A., On the extremal properties of the average eccentricity. Comput. Math. Appl., 2012, 64, 2877-2885.

Imran M., Siddiqui M.K., Abunamous A.A.E., Ali D., Rafique S.H., Baig A.Q., Eccentricity based topological indices of an Oxide network. Mathematics, 2018, 6, No. 126.

Imran M., Baig A.Q., Azhar M.R., Farahani M.R., Zhang X., Eccentricity based geometric-arithmetic and atom-bond connectivity indices of copper oxide CuO. Int. J. Pure Appl. Math., 2017, 117, 481-502.

Klavžar S., Gutman I., A comparison of the Schultz molecular topological index with the Wiener index. J. Chem. Inf. Comput. Sci., 1996, 36, 1001-1003.

Liu J.-B., Javaid M., Awais H.M., Zagreb indices of generalized operations on graphs. IEEE Access, 2019, 7, 105479-105488.

Rucker G., Rucker C., On topological indices, boiling points, and cycloalkanes. J. Chem. Inf. Comput. Sci., 1999, 39, 788-802.

Sardana S., Madan A.K., Predicting anti-HIV activity of TIBO derivatives: Acomputational approach using a novel topological descriptor. J. Mol. Model., 2002, 8, 258-265.

Sharma V., Goswami R., Madan A.K., Eccentric connectivity index: A novel highly discriminating topological descriptor for structure property and structure activity studies. J. Chem. Inf. Comput. Sci., 1997, 37, 273-282.

Tang J.-H., Ali U., Javaid M., Shabbir K., Zagreb connection indices of subdivision and semi-total point operations on graphs. J. Chem., 2019, 1-14, Article ID: 9846913.

Tang Y., Zhou B., On average eccentricity. MATCH-Commun. Math. Co., 2012, 67, 405-423.

Vukiĉević D., Graovac A., A note on the comparison of the first and second normalized Zagreb eccentricity indices. Acta Chim. Slov., 2010, 57, 524-528.

Wiener H., Correlation of heats of isomerization and differences in heats of vaporization of isomers, among the paraffin hydrocarbons. J. Am. Chem. Soc., 1947, 69, 17-20.

Yu G., Feng L., On connective eccentricity index of graphs. MATCH-Commun. Math. Co., 2013, 69, 611-628.

Zahid M.A., Baig A.Q., Naeem M., Azhar M.R., Eccentricity based topological indices of a cyclic octahedron structure. Mathematics, 2018, 6, No. 141.

Zheng L., Wang Y., Gao W., Topological indices of Hyaluronic Acid-Paclitaxel conjugates molecular structure in cancer treatment. Open Chem., 2019, 17, 81-87.

Zhang X., Baig A.Q., Azhar M.R., Farahani M.R., Imran M., The average eccentricity and eccentricity based geometric-arithmetic index of tetra sheets. Int. J. Pure Appl. Math., 2017, 116, 467-479.

Zhang H., Zhang F., The Clar covering polynomial of hexagonal systems. Discrete Appl. Math., 1996, 69, 147-167. 